\title{
Desempenho de Coelhos até a Desmama de Acordo com o Tamanho da Ninhada e o Nível de Amido nas Dietas
}

\author{
Haroldo Garcia de Faria ${ }^{1}$, Cláudio Scapinello ${ }^{2}$, Rosane Marina Peralta ${ }^{3}$, Thierry Gidenne ${ }^{4}$, \\ Antonio Carlos Furlan², Marcia Aparecida Andreazzi ${ }^{5}$
}

\begin{abstract}
RESUMO - Foi conduzido um experimento com o objetivo de estudar as influências do padrão alimentar de coelhos durante a fase de lactação, em função do nível de amido nas dietas e da disponibilidade de leite modulada pelo tamanho da ninhada, sobre o desempenho de láparos até a desmama (35 dias). Cem ninhadas foram mantidas com oito láparos ao nascer até o 16ํㅜ dia de vida. A partir do 16ํㅜ dia de idade até a desmama, 60 ninhadas foram reduzidas para quatro láparos, enquanto as demais foram mantidas com oito, recebendo dietas contendo nível alto (24\%) ou normal (15\%) de amido. As ninhadas com quatro láparos apresentaram maior ganho de peso diário, independentemente dos intervalos analisados, sendo desmamados com maior peso em relação aos mantidos em ninhadas mais numerosas. Este efeito também foi observado naqueles que receberam dietas com alto teor de amido somente a partir do $24 \underline{0}$ dia de lactação. Os láparos mantidos em ninhadas mais numerosas apresentaram maior consumo diário de ração até $26^{0}$ dia de idade. À medida que os láparos mantidos em ninhadas menores apresentaram superioridade no crescimento e a disponibilidade de leite pela coelha foi diminuída pela proximidade do final da lactação, o consumo de ração na fase final de lactação passou a ser maior para os láparos mantidos em ninhadas menores. Por outro lado, a dieta mais rica em amido proporcionou maior consumo diário, independentemente do intervalo analisado. O consumo individual de leite foi maior para láparos mantidos em ninhadas menores, não havendo diferença em relação aos níveis de amido nas dietas disponibilizadas aos animais.
\end{abstract}

Palavras-chave: desempenho, ingestão de leite, ingestão de ração, lactação, láparos

\section{Performance of Rabbits During Lactation Phase According to the Litter Size and Starch Level in the Diets}

\begin{abstract}
The experiment was carried out to study the influences of the feeding pattern of rabbits during the lactation phase according function of the starch level in the diets and availability of milk modulated by litter size on performance young rabbits before weaning (35 days). One hundred litters were maintained to eight rabbits at birth until the 16 day old. From 16 to 35 days old, 60 litter were reduced for four young rabbits, while the others were maintained with eight young rabbits, feeding with high (24\%) or normal (15\%) starch level. The litters with four young rabbits presented higher daily weight gain, independently of the analyzed intervals, and were weaned with higher weight than young rabbits maintained in more numerous litters. This effect was also observed only for young rabbits feeding diets with high starch level, from 24 days to weaning. The young rabbits maintained in more numerous litters presented the higher daily consumption of ration until 26 days of age. As young rabbits maintained in smaller litter presented superiority in the growth and the availability of milk for the does was decreased by the proximity of the end lactation, the feed intake in the final phase of lactation became the higher for the young rabbits maintained in smaller litters. The diet with high level starch showed higher feed intake, independently of the analyzed interval. The individual milk intake was the higher for young rabbits maintained in smaller litters, but no difference was observed in relation to the starch levels in diets.
\end{abstract}

Key Words: feed intake, lactation, milk intake, performance, young rabbits

\section{Introdução}

Embora exista grande número de publicações sobre a fisiologia de animais domésticos, o coelho é uma das espécies cujo conhecimento sobre suas características fisiológicas, principalmente do sistema digestivo, permanece incompleto. Desordens digestivas, observadas freqüentemente em coelhos durante a fase de amamentação e logo após a desmama, podem estar relacionadas com o padrão alimentar (qualidade e quantidade) e a maturação dos processos digestivos (Gidenne \& Perez, 1993).

\footnotetext{
${ }^{1}$ Zootecnista do Biotério Central - UEM - Av. Colombo 5790, Maringá - PR, CEP: 87020-900 (haroldogf@wnet.com.br)

${ }^{2}$ Professor do Departamento de Zootecnia UEM - Av. Colombo 5790, Maringá - PR, CEP: 87020-900.

3 Professora do Departamento de Bioquímica - UEM - Av. Colombo 5790, Maringá - PR, CEP: 87020-900.

${ }^{4}$ Pesquisador do INRA-Institut National de La Recherche Agronomique - Tolouse - França.

${ }^{5}$ Professora do curso de Medicina Veterinária - CESUMAR - Av. Guerdner 1660, Maringá - PR, CEP: 87050-390
} 
A disponibilidade de leite para os láparos, modulada pelo número de filhotes da ninhada, número de mamadas por dia e produção de leite pelas fêmeas, interfere no início do consumo de alimentos sólidos e determina tanto o início da ingestão de alimento sólido, como o volume ingerido.

Publicações a respeito do comportamento alimentar de coelhos antes da desmama limitam-se, em sua maioria, em demonstrar o consumo de leite, mas poucas relacionam este comportamento com a ingestão de alimentos sólidos (Lebas, 1970; Mcnitt \& Moody, 1988). Maertens \& De Groote (1990), estudando o comportamento alimentar de láparos antes da desmama, concluíram que o consumo precoce de alimentos sólidos pode estimular a secreção enzimática pancreática, resultando em decréscimo da mortalidade após a desmama.

Por outro lado, Scapinello et al. (1999), estudando a capacidade digestiva de coelhos durante o período após a desmama, de acordo com o padrão de consumo leite/alimento sólido antes da desmama, concluíram que a menor ingestão individual de leite e consumo mais precoce de alimentos sólidos, de láparos mantidos em ninhadas mais numerosas, não conduziram a alterações na capacidade de digestão de carboidratos solúveis após a desmama, avaliadas pela atividade das enzimas amilase e maltase no jejuno e íleo.

Entre as dificuldades para estudar o comportamento alimentar de coelhos jovens, em condições normais de manejo, salienta-se o fato destes permanecerem com a fêmea até o desmame, usando o mesmo comedouro e consumindo a mesma ração da matriz. Dessa forma, a determinação do tempo exato do início e a quantidade de ingestão de alimentos sólidos pelos láparos exigem o uso de equipamentos que permitam o acesso exclusivo ou a separação dos láparos da fêmea.

Segundo Mcnitt \& Moody (1988), fica evidente a importância da ingestão de alimentos sólidos durante o período de amamentação, uma vez que os láparos, a partir dos 15 a 20 dias de idade, não têm suas exigências atendidas para alcançar taxas de crescimento máximo somente com a ingestão de leite.

Por outro lado, mudanças na composição das dietas, particularmente o aumento da ingestão de amido, alteram a natureza da digesta que chega ao ceco, com conseqüentes mudanças nos processos de fermentação. A possibilidade da incompleta digestão do amido, associada à imaturidade do sistema enzimático do trato digestivo de coelhos jovens, pode representar aumento no fluxo de carboidratos solúveis para o ceco, alterando a atividade microbiana e aumentando os riscos de distúrbios digestivos (Cheeke \& Patton, 1980; Blas et al. 1994).

Este estudo foi conduzido com o objetivo de estudar as influências da disponibilidade de leite, modulada pelo tamanho da ninhada, e o nível de amido em dietas sobre o desempenho e consumo de leite/ alimentos sólidos de láparos até a desmama.

\section{Material e Métodos}

Para modular o padrão de consumo de leite e ração antes da desmama, 100 ninhadas, obtidas de fêmeas da raça Nova Zelândia Branco, de segundo parto, foram eqüalizadas para oito láparos do nascer até o $16^{0}$ dia de vida. A partir do $16^{0}$ dia de idade, até a desmama, aos 35 dias de idade, as ninhadas foram submetidas à alta e baixa disponibilidades de leite, variando o número de láparos, sendo 60 ninhadas reduzidas para quatro láparos (L4), enquanto as demais foram mantidas com oito láparos (L8).

As ninhadas foram distribuídas em um esquema fatorial 2 x 2 (dois tamanhos de ninhadas versus dois níveis de amido nas rações), alojadas em gaiolas de arame galvanizado, providas de bebedouro automático e comedouro semi-automático de chapa galvanizada, localizadas em galpão de alvenaria, piso em alvenaria, paredes laterais de $50 \mathrm{~cm}$ e o restante em tela e cortina de plástico para controle de ventos.

As dietas contendo níveis normal e alto de amido (Tabela 1) foram peletizadas a seco e fornecidas à vontade, juntamente com água, a partir do 16으 dia.

Os ninhos com os láparos permaneceram, desde o nascimento, em gaiolas separadas, ao lado das fêmeas e a amamentação dos filhotes ocorreu uma vez ao dia, pela manhã, quando, em seguida, as fêmeas retornavam para suas gaiolas de origem.

Os láparos foram pesados no início do experimento, aos 16 dias e, em seguida, a cada três dias até a desmama, aos 35 dias de idade. As pesagens foram feitas antes e depois da amamentação com o objetivo de observar o consumo de leite da ninhada pela diferença de peso. As rações fornecidas e as sobras também foram pesadas logo após a pesagem dos animais.

Os resultados foram submetidos à análise de variância, utilizando-se o programa SAEG (UFV, 1997) e o modelo a seguir:

$$
\mathrm{Y}_{\mathrm{ijK}}=\mu+\mathrm{N}_{\mathrm{i}}+\mathrm{A}_{\mathrm{j}}+\mathrm{NA}_{\mathrm{ij}}+\mathrm{e}_{\mathrm{ijk}}
$$


Tabela 1 - Composição percentual das dietas experimentais

Table 1 - Percent and chemical composition of the experimental diets

\begin{tabular}{|c|c|c|c|}
\hline $\begin{array}{l}\text { Ingredientes } \\
\text { Ingredient }\end{array}$ & $\begin{array}{l}\text { Unidade } \\
\text { Unit }\end{array}$ & $\begin{array}{l}\text { Amido normal } \\
\text { Normal starch }\end{array}$ & $\begin{array}{l}\text { Alto amido } \\
\text { High starch }\end{array}$ \\
\hline $\begin{array}{l}\text { Milho } \\
\text { Corn }\end{array}$ & $\mathrm{kg}$ & 14,110 & 32,500 \\
\hline $\begin{array}{l}\text { Farelo de soja } \\
\text { Soybean meal }\end{array}$ & $\mathrm{kg}$ & 12,600 & 16,000 \\
\hline $\begin{array}{l}\text { Farelo de trigo } \\
\text { Wheat meal }\end{array}$ & $\mathrm{kg}$ & 25,000 & 9,500 \\
\hline $\begin{array}{l}\text { Feno de alfafa } \\
\text { Alfalfa hay }\end{array}$ & $\mathrm{kg}$ & 28,000 & 27,000 \\
\hline $\begin{array}{l}\text { Feno de aveia } \\
\text { Oats hay }\end{array}$ & $\mathrm{kg}$ & 15,520 & 9,000 \\
\hline $\begin{array}{l}\text { Sal comum } \\
\text { Common salt }\end{array}$ & $\mathrm{kg}$ & 0,400 & 0,400 \\
\hline $\begin{array}{l}\text { Fosfato bicálcico } \\
\text { Dicalcium phosphate }\end{array}$ & $\mathrm{kg}$ & 0,100 & 0,340 \\
\hline $\begin{array}{l}\text { Calcário } \\
\text { Limestone }\end{array}$ & $\mathrm{kg}$ & 0,700 & 0,700 \\
\hline $\begin{array}{l}\text { DL-Metionina } \\
\text { DL-Methionine }\end{array}$ & $\mathrm{kg}$ & 0,070 & 0,060 \\
\hline $\begin{array}{l}\text { Mist. Vit+Min }{ }^{1} \\
{\text { Premix vit }+ \text { min. }^{1}}\end{array}$ & $\mathrm{~kg}$ & 0,500 & 0,500 \\
\hline $\begin{array}{l}\text { Óleo vegetal } \\
\text { Vegetable oil }\end{array}$ & $\mathrm{kg}$ & 3,000 & - \\
\hline $\begin{array}{l}\text { Casca de arroz } \\
\text { Rice hulls }\end{array}$ & $\mathrm{kg}$ & - & 4,000 \\
\hline Total & $\mathrm{kg}$ & 100,00 & 100,00 \\
\hline
\end{tabular}

Composição analisada com base na matéria natural

Analyzed composition in natural matter

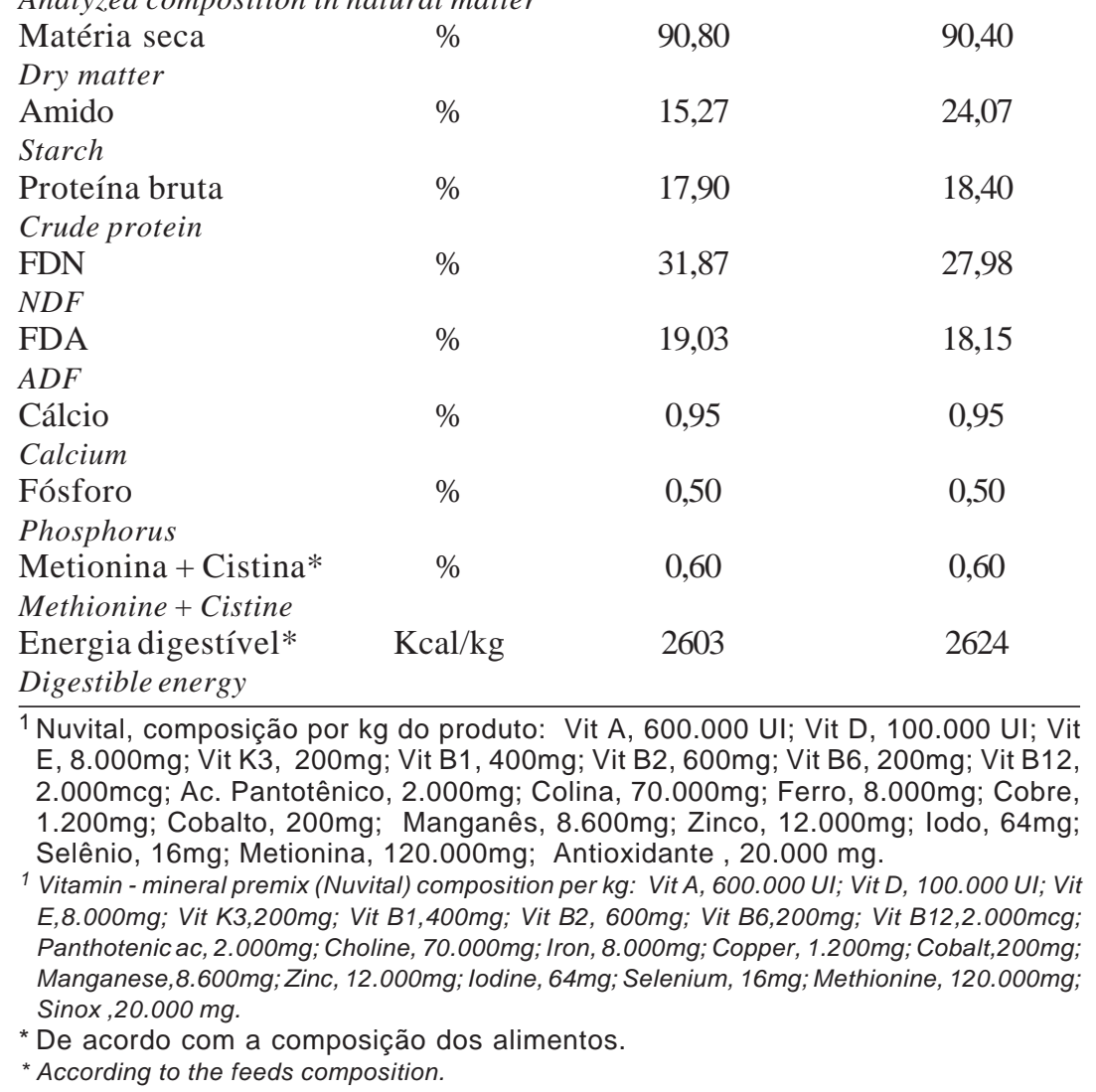


em que $\mathrm{Y}_{\mathrm{ijk}}=$ observação relativa ao indivíduo $k$, recebendo o nível $j$ de amido na ração e mantido em ninhadas com número $i$ de láparos; $\mu$ = média geral de cada característica; $\mathrm{N}_{\mathrm{i}}=$ efeito do tamanho da ninhada $i$, sendo $i_{1}=$ ninhadas com quatro láparos, $i_{2}=$ ninhadas com oito láparos; $A_{j}=$ efeito do nível de amido nas dietas, sendo $j_{1}=15 \%$ de amido e $j_{2}=24 \%$ de amido; $\mathrm{NA}_{\mathrm{ij}}=$ interação entre o tamanho da ninhada $i$ até a desmama e o nível de amido $j$ na ração; $\mathrm{e}_{\mathrm{ijk}}=$ erro aleatório associado a cada observação.

Para comparação das médias, foi utilizado o teste de $\mathrm{F}$ a $5 \%$.

\section{Resultados e Discussão}

Não foi observada interação $(P>0,05)$ entre o tamanho da ninhada e o nível de amido nas dietas para o ganho de peso diário dos láparos em qualquer dos intervalos até o desmame e para o peso vivo aos 35 dias de idade (Tabela 2).

As ninhadas com quatro láparos apresentaram maior ganho de peso diário $(\mathrm{P}<0,05)$, independentemente dos intervalos de pesagem. Portanto, os animais foram desmamados com maior peso em relação àqueles mantidos em ninhadas com oito.

Por outro lado, a dieta rica em amido proporcionou maior ganho de peso diário $(\mathrm{P}<0,06)$ aos láparos a partir do intervalo de 27 a 29 dias de idade, independentemente do tamanho da ninhada, ocasio- nando maior peso vivo ao desmame.

Com relação ao consumo médio diário de ração, não foi observada interação $(\mathrm{P}>0,05)$ entre o tamanho da ninhada e o nível de amido nas dietas. As ninhadas com quatro láparos tiveram menor consumo até os 26 dias de idade, em relação à com oito láparos, salientando-se que, a partir dos 30 dias de idade, os láparos mantidos em ninhadas menores passaram a consumir mais ração $(\mathrm{P}<0,05)$ até a desmama (35 dias de idade), possivelmente em razão do maior desenvolvimento destes animais. Considerando o período total avaliado não houve diferença $(\mathrm{P}>0,05)$ no volume de ração ingerida pelos láparos, independentemente do tamanho da ninhada. A dieta rica em amido também proporcionou maior consumo $(\mathrm{P}<0,05)$ de ração em todos os intervalos observados (Tabela 3).

Uma importante situação a ser abordada é a quantidade de vezes que as fêmeas tiveram acesso aos ninhos para amamentar os láparos. No presente trabalho, optou-se por um único acesso diário. Trabalhos realizados com coelhos de tipo carne por Mcnitt \& Moody (1988) e Gyarmati et al. (2000) apresentaram claras evidências de que coelhos jovens tendem a ser amamentados duas vezes ao dia, tendo acesso a maiores quantidades de nutrientes em relação aos que foram amamentados só uma vez ao dia e, como conseqüência, tiveram menor velocidade de crescimento durante o período após a desmama.

Tabela 2 - Ganho de peso diário e peso vivo ao desmame (PV35) de láparos, em diferentes intervalos, durante a fase de lactação, em função do nível de amido nas dietas e da disponibilidade de leite modulada pelo tamanho da ninhada (quatro ou oito láparos)

Table 2 - Means of daily weight gain and live weight 35 days of the young rabbits in different intervals during lactation phase, according to the dietary starch levels (high [24\%] or normal [15\%]) and the milk availability modulated by litter size (four or eight young rabbits)

\begin{tabular}{|c|c|c|c|c|c|c|c|c|}
\hline \multirow[b]{2}{*}{$\begin{array}{l}\text { Período/dias } \\
\text { Period/days }\end{array}$} & \multicolumn{2}{|c|}{$\begin{array}{c}\text { Tamanho da } \\
\text { ninhada } \\
\text { Litter size }\end{array}$} & \multicolumn{2}{|c|}{$\begin{array}{l}\text { Nível de amido } \\
\text { Starch level (\%) }\end{array}$} & \multirow[b]{2}{*}{$\begin{array}{l}\text { Média } \\
\text { Mean }\end{array}$} & \multirow[b]{2}{*}{ CV(\%) } & \multicolumn{2}{|c|}{$\begin{array}{l}\text { Significância }^{1} \\
\text { Significance }\end{array}$} \\
\hline & $\mathrm{L} 4$ & L8 & 15 & 24 & & & $\begin{array}{l}\text { Ninhada } \\
\text { Litter }\end{array}$ & $\begin{array}{l}\text { Amido } \\
\text { Starch }\end{array}$ \\
\hline $16-20$ & 24,0 & 12,3 & 18,4 & 17,8 & 18,1 & 21,0 & 0,01 & Ns \\
\hline $21-23$ & 24,2 & 15,7 & 19,4 & 20,5 & 19,9 & 29,2 & 0,01 & Ns \\
\hline $24-26$ & 25,8 & 21,2 & 22,4 & 24,5 & 23,5 & 23,0 & 0,01 & Ns \\
\hline $27-29$ & 39,1 & 33,0 & 34,4 & 37,7 & 36,1 & 14,7 & 0.01 & 0,01 \\
\hline 30-32 & 45,3 & 36,2 & 39,6 & 41,8 & 40,7 & 16,7 & 0,01 & 0,02 \\
\hline 33-35 & 47,7 & 40,5 & 42,4 & 45,8 & 44,1 & 17,7 & 0,01 & 0,04 \\
\hline PV35(g) & 851 & 695 & 751 & 795 & 773 & 10,0 & 0,01 & 0,01 \\
\hline
\end{tabular}

${ }^{1}$ Diferem $(\mathrm{P}<0,05)$ pelo teste de $\mathrm{F}($ Differ $[P<.05]$ by $F$ test). 
Debray et al. (2000), analisando a eficiência digestiva de coelhos, antes e após a desmama, alimentados com dietas contendo duas relações amido/ fibra (15,1 e 12,5\% de amido e 30,1 e 34,2\% de FDN, respectivamente), provenientes de ninhadas constituídas de nove láparos, concluíram que a eficiência alimentar dos coelhos foi reduzida quando alimentados com dietas contendo baixo amido/alta fibra, antes e após a desmama. Os autores também constataram que o efeito da relação amido/fibra e a regulação da ingestão antes da desmama devem ser mais profundamente estudados.

Não foi possível mensurar o consumo de alimento (leite/ração) individual dos láparos e, com isso, calcular a variabilidade entre os indivíduos. Até os 20 dias de idade, os coelhos consumiram exclusivamente leite e, a partir desta idade, começaram a ingerir alimento sólido. Estes resultados estão de acordo com os de Maertens \& De Groote (1990), que também observaram consumo de alimento sólido muito baixo antes de 22-23 dias de idade.

Verificou-se que os láparos mantidos em ninhadas menores, apesar da maior disponibilidade de leite, consumiram mais ração a partir de 29 dias de idade que os mantidos em ninhadas maiores. Este fato ocorreu em razão do maior crescimento dos láparos deste grupo, o que levou ao aumento de suas necessidades de nutrientes.
Scapinello et al. (1999), verificando a capacidade digestiva de coelhos (16-32 dias) provenientes de ninhadas com 10 e quatro láparos, constataram maior consumo médio de leite e de ração, bem como maior ganho de peso, em ninhadas de quatro animais. Os autores afirmam que, em condições que levam à menor disponibilidade individual de leite, a ingestão de alimento sólido ocorre mais precocemente, mudando a relação de ingestão leite/alimento sólido, particularmente na fase em que os láparos iniciam a ingestão de ração, fato também observado neste experimento.

Zomborszky-Kovacs et al. (2000) afirmam que problemas relacionados a distúrbios digestivos após o desmame são mais comuns em coelhos jovens amamentados duas vezes ao dia. Os autores também concluem que coelhos amamentados duas vezes ao dia apresentam pior desempenho em relação aos amamentados uma vez ao dia. Contudo, Mcnitt \& Moody (1988), verificando a ingestão de leite e a taxa de crescimento de coelhos em amamentação, constataram que animais que consomem mais leite durante a fase de amamentação apresentam maior peso e maior crescimento após a desmama.

De modo geral, o que se verifica é que, quanto mais cedo iniciar a ingestão de alimento sólido antes da desmama, menos problemas digestivos terão estes animais após a desmama. Padilha et al. (1996), estudando a microflora cecal e o padrão de fermen-

Tabela 3 - Consumo de ração diário de láparos (g/dia), em diferentes intervalos, durante a fase de lactação, em função do nível de amido nas dietas e da disponibilidade de leite modulada pelo tamanho da ninhada (quatro ou oito láparos)

Table 3 - Daily feed intake of young rabbits $(g / d)$ in different intervals, during the lactation phase, according to the dietary starch levels (high [24\%] or normal [15\%]) and the milk availability modulated by litter size (four or eight young rabbits)

\begin{tabular}{|c|c|c|c|c|c|c|c|c|}
\hline \multirow[b]{2}{*}{$\begin{array}{l}\text { Período/dias } \\
\text { Period/days }\end{array}$} & \multicolumn{2}{|c|}{$\begin{array}{c}\text { Tamanho da } \\
\text { ninhada } \\
\text { Litter size }\end{array}$} & \multicolumn{2}{|c|}{$\begin{array}{l}\text { Nível de amido } \\
\text { Starch level (\%) }\end{array}$} & \multirow[b]{2}{*}{$\begin{array}{l}\text { Média } \\
\text { Mean }\end{array}$} & \multirow[b]{2}{*}{ CV(\%) } & \multicolumn{2}{|c|}{$\begin{array}{l}\text { Significância }^{1} \\
\text { Significance }\end{array}$} \\
\hline & L4 & L8 & 15 & 24 & & & $\begin{array}{c}\text { Ninhada } \\
\text { Litter }\end{array}$ & $\begin{array}{l}\text { Amido } \\
\text { Starch }\end{array}$ \\
\hline $16-20$ & - & - & - & - & - & - & - & - \\
\hline $21-23$ & 1,2 & 2,3 & 1,2 & 2,3 & 1,76 & 66,0 & 0,01 & 0,01 \\
\hline $24-26$ & 10,7 & 13,1 & 10,3 & 13,5 & 11,9 & 42,8 & 0,01 & 0,01 \\
\hline $27-29$ & 26,4 & 28,5 & 24,8 & 30,1 & 27,4 & 21,9 & 0,10 & 0,01 \\
\hline $30-32$ & 43,5 & 37,7 & 37,0 & 44,2 & 40,6 & 24,6 & 0,01 & 0,01 \\
\hline $33-35$ & 55,4 & 47,2 & 47,4 & 55,1 & 51,3 & 19,4 & 0,01 & 0,01 \\
\hline $20-35$ & 27,4 & 25,7 & 24,2 & 29,0 & 26,6 & 18,6 & 0,10 & 0,01 \\
\hline
\end{tabular}

${ }^{1}$ Diferem $(P<0,05)$ pelo teste $F($ Differ $[P<.05]$ by $F$ test $)$. 
Tabela 4 - Consumo de leite (g/láparos) no período de 16 a 35 dias de idade, em função do nível de amido nas dietas e da disponibilidade de leite modulada pelo tamanho da ninhada (quatro ou oito láparos)

Table 4 - Milk intake (g/young rabbits) from 16-35 days of age, according to the dietary starch levels (high [24\%] or normal [15\%]) and the milk availability modulated by litter size (four or eight young rabbits)

\begin{tabular}{|c|c|c|c|c|c|c|c|c|}
\hline \multirow{3}{*}{$\begin{array}{l}\text { Período/dias } \\
\text { Period/days }\end{array}$} & \multicolumn{2}{|c|}{$\begin{array}{c}\text { Tamanho da } \\
\text { ninhada } \\
\text { Litter size }\end{array}$} & \multicolumn{2}{|c|}{$\begin{array}{l}\text { Nível de amido } \\
\text { Starch level (\%) }\end{array}$} & \multirow{3}{*}{$\begin{array}{l}\text { Média } \\
\text { Mean }\end{array}$} & \multirow{3}{*}{$\begin{array}{l}C V(\%) \\
C V(\%)\end{array}$} & \multicolumn{2}{|c|}{$\begin{array}{l}\text { Significância }^{1} \\
\text { Significance }\end{array}$} \\
\hline & L4 & L8 & 15 & 24 & & & Ninhada & Amido \\
\hline & $L 4$ & $L 8$ & 15 & 24 & & & Litter & Starch \\
\hline 16 & 40,3 & 23,5 & 31,5 & 32,3 & 31,9 & 20,0 & 0,01 & Ns \\
\hline 20 & 40,5 & 23,4 & 33,1 & 32,7 & 32,9 & 15,8 & 0,01 & Ns \\
\hline 23 & 38,5 & 25,4 & 32,5 & 31,4 & 31,9 & 17,0 & 0,01 & Ns \\
\hline 26 & 38,7 & 22,5 & 31,4 & 29,8 & 30,6 & 19,4 & 0.01 & Ns \\
\hline 29 & 36,0 & 20,3 & 28,7 & 27,7 & 28,2 & 21,1 & 0,01 & Ns \\
\hline 32 & 30,9 & 16,7 & 24,8 & 22,8 & 23,8 & 29,3 & 0,01 & Ns \\
\hline 35 & 27,3 & 15,1 & 22,7 & 19,5 & 21,2 & 37,2 & 0,01 & 0,06 \\
\hline
\end{tabular}

${ }^{1}$ Diferem $(P<0,05)$ pelo teste de $F($ Differ $[P<.05]$ by $F$ test $)$.

tação em coelhos jovens alimentados exclusivamente com leite, concluíram que a ingestão de sólido é o fator principal para o estabelecimento da flora fibrolítica e do padrão de fermentação cecal. Portanto, é de interesse estimular a ingestão de alimento sólido, em coelhos jovens, o mais cedo possível antes da desmama para promover o estabelecimento da flora simbiótica.

Não foram observados problemas digestivos nos láparos que receberam dieta contendo alto nível (24\%) de amido. Estes resultados são conflitantes com os de Blas et al. (1994), segundo os quais dietas com alto teor de amido são uma das possíveis causas de problemas digestivos antes e após a desmama. Maertens (1992) também mencionou que, para evitar problemas digestivos, níveis superiores a $15 \%$ de amido na dieta não são recomendáveis para coelhos jovens.

\section{Conclusões}

Não houve interação entre o tamanho da ninhada e o nível de amido nas dietas sobre o desempenho dos láparos em nenhum dos períodos estudados. O nível de amido não influenciou o consumo de leite para os láparos no período de 16-35 dias de idade.

A dieta com alto nível de amido (24\%) proporcionou maior ganho de peso diário a partir de 24 dias de idade e maior consumo diário de ração em todos os períodos analisados.

Por outro lado, a maior disponibilidade individual de leite para láparos mantidos em ninhadas menores proporcionou maior desempenho dos animais, no período de 16 a 35 dias de idade, em relação àqueles mantidos em ninhadas com oito láparos.

\section{Literatura Citada}

BLAS, E.; CERVERA, C.; CARMONA, F.J. Effect of two diets with varied starch and fibre levels on the performances of 4-7 weeks old rabbits. World Rabbit Science, v.2, n.1, p.117-121, 1994.

CHEEKE, P.R.; PATTON, N.M. Carbohydrate-overload of the hindgut. A probable cause of enteritis. Journal Applied Rabbit Research, v.3, n,3, p.20-23, 1980.

DEBRAY. L.; GIDENNE, T.; FORTUN-LAMOTHE, L. et al. Digestive efficiency before and after weaning, according to the dietary starch/fibre ratio. In: WORLD RABBIT CONGRESS, 7., 2000, Valencia. Proceedings... Valencia: 2000. p.167-174.

GIDENNE, T.; PEREZ, J. M. Effect of dietary starch origin on digestión in the rabbit. 1. Digestibility measurements from weaning to slaughter. Animal Feed Science and Technology, v.42, n.3-4, p.237-242, 1993.

GYARMATI, T.; SZENDRO, Z.S.; MAERTENS, L. et al. Effect of suckling twice a day on the performance of suckling and growing rabbits. In: WORLD RABBIT CONGRESS, 7., 2000, Valencia. Proceedings... Valencia: 2000. p.283-290.

LEBAS, F. Alimentation et croissance du laperau sous la mère. Recueil Médicine Vétérinaire de l'Ecole d'Alfort, v.146, p.1065-1070, 1970 .

MAERTENS, L.; De GROOTE, G. Feed intake of rabbit kit before weaning and attempts to increase it. Journal Applied Rabbit Research, v.13, p.151-158, 1990.

MAERTENS, L. Rabbit nutrition and feeding: a review of recent developments. Journal of Applied Rabbit Research, v.13, p.889-913, 1990.

R. Bras. Zootec., v.33, n.4, p.894-900, 2004 
Mc NITT, J.I.; MOODY, J. Milk intake and growth rates of sucking rabbits. Journal of Applied Rabbit Research, v.11, p.117-119, 1988.

PADILHA, M.T.S.; LICOIS, D.; GIDENNE, T. et al. Caecal microflora and fermentation pattern in exclusively milkfed young rabbits. In: WORLD RABBIT CONGRESS, 6., 1996, Toulouse. Proceedings... Tolouse: AFC, 1996. p.247-250.

SCAPINELLO, C.; GIDENNE, T.; LAMONTHE, L. F. Digestive capacity of rabbit during the post-weaning period, according to the milk/solid feed intake pattern before weaning. Reproduction Nutrition Development, v.39, n.4, p.423-432, 1999.
UNIVERSIDADE FEDERAL DE VIÇOSA - UFV. SAEG Sistema de análises estatísticas e genéticas. Versão 7.1. Viçosa, MG: 1997. 150p. (Manual do usuário)

ZOMBORSKY-KOVÁC, M.; GYARMATI, T.; PARIZS, T. et al. Some physiological properties of the digestive tract in traditionally reared and exclusively milk-fed young rabbits. In: WORLD RABBIT CONGRESS, 7., 2000, Valencia. Proceedings ... Valencia: 2000. p.499-506.

Recebido em: 22/04/03

Aceito em: 09/09/03 